\title{
Attempt of an Axiomatic Foundation of Quantum Mechanics and More General Theories. III
}

GÜNTHER LUDWIG

Institut für Theoretische Physik (I), Universität Marburg

Received March 6, 1968

\begin{abstract}
Starting from axioms as physical as possible [1, 2, 3] about "effects" and "ensembles", we shall investigate further consequences.

Concerning part I and II $[4,5]$ the axioms can be so formulated as to be surveyed more easily.

Besides, it is possible to prove some important theorems more simply.

New structures of the lattice of decision effects are pointed out, leading in two subsequent papers at last to the final aim, the structure of Hilbert-space.
\end{abstract}

\section{Summary of Former Results}

After the publication of part II of this exposition it has turned out that the axioms for a foundation can be formulated still somewhat more skilfully (at least in the case of a finite-dimensional ensemblespace, see below). They can be so extended that, in fact, (finite-dimensional) Hilbert-spaces over the fields of the real or complex numbers or of the quaternions remain as irreducible solutions of the system of axioms. Therefore the axioms shall here be briefly made up once more. A physically heuristic argument can be found in $[1,2,3]$. Physical arguments more detailed will be given in the second edition of the book „,Die Grundlagen der Quantenmechanik“.

The starting point of our foundation are the sets $\underline{K}$ of the ensembles $\underline{V}$ and $\underline{L}$ of the effects $\underline{F}$ and a probability function $\mu$ on $\underline{K} \times \underline{L}$ satisfying:

Axiom 1.

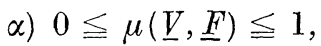

ק) $\mu\left(\underline{V}_{1}, \underline{F}\right)=\mu\left(\underline{V}_{2}, \underline{F}\right)$ for all $\underline{F} \in \underline{L}$ implies $\underline{V}_{1}=\underline{V}_{2}$,

r) $\mu\left(\underline{V}, \underline{F}_{1}\right)=\mu\left(\underline{V}, \underline{F}_{2}\right)$ for all $\underline{V} \in \underline{K}$ implies $\underline{F}_{1}=\underline{F}_{2}$,

$\delta$ ) there exists $\underline{F}_{0} \in \underline{L}$ (denoted by 0$)$ with $\mu(\underline{V}, 0)=0$ for all $\underline{V} \in \underline{K}$,

ع) for each $\underline{V} \in \underline{K}$ there exists $\underline{F} \in \underline{L}$ with $\mu(\underline{V}, \underline{F})=1$.

By the set of all functions on $\underline{L} X(\underline{F}):=\sum_{i=1}^{n} a_{i} \cdot \mu\left(\underline{V}_{i}, \underline{F}\right)$ with $\underline{V}_{i} \in \underline{K}, a_{i}$ real numbers and $n$ any finite integer, a real linear space $B$ is defined. We will only pursue the case where $B$ is finite-dimensional. By $X(\underline{F})=\mu(\underline{V}, \underline{F}), \underline{K}$ can be indentified with a subset of $B$. By $\|X\|$ : $=\sup \{|X(\underline{F})| \mid \underline{F} \in \underline{L}\}$ for all $X \in B, B$ becomes a normed linear space.

1 Commun. math. Phys., Vol. 9 
Definition 1. $K$ denotes the closed convex hull of $\underline{K}$ in $B$.

By $\mu(X, \underline{F}):=X(\underline{F})$ the definition of $\mu$ can be extended to the entire $B \times \underline{L}$. If $\underline{F}$ is fixed, then by $\mu(X, \underline{F})$ a linear functional is defined over $B$ so that $\underline{L}$ can be identified with a subset of dual space $B^{\prime}$ of $B$.

Definition 2. a) $L$ denotes closure of $\underline{L}$ in $B^{\prime}$.

b) $\hat{\bar{L}}$ denotes the closure of the set $\left\{Y \mid Y \in B^{\prime}, Y=\lambda F, \lambda \geqq 0, F \in \hat{L}\right.$ and $\lambda \mu(V, F) \leqq 1$ for all $\left.V \in K\right\}$, where $\hat{L}$ denotes the closed convex hull of $\underline{L}$ in $B^{\prime}$.

Thus $\hat{\bar{L}}$ is convex as well. By the definition

$$
Y_{1} \leqq Y_{2} \text { iff } \mu\left(V, Y_{1}\right) \leqq \mu\left(V, Y_{2}\right) \text { for all } V \in K,
$$

$B^{\prime}$ becomes a partially ordered vector space. We formulate the first principal law about measurement in two parts:

Axiom 2a $\mathbf{a}^{1}$. For each pair $F_{1} \in L, F_{2} \in L$ there exists $F_{3} \in L$ so that

$$
F_{1} \leqq F_{3}, \quad F_{2} \leqq F_{3}
$$

and for each $V \in K$ with $\mu\left(V, F_{1}\right)=0$ and $\mu\left(V, F_{2}\right)=0, \mu\left(V, F_{3}\right)=0$ holds too.

This axiom can be illustrated by two filters $F_{1}$ and $F_{2}$ (see [1], p. 1314 p.p.). If we very frequently connect the filters $F_{1}, F_{2}$ in the form of a filter packet $F_{1} F_{2} F_{1} F_{2} F_{1} F_{2} \ldots$, we (approximatively) obtain the $F_{3}$ desired.

Without proof, we now summarize some conclusions of the axioms 1 and $2 \mathrm{a}$ drawn in $[4,5]$. An extremal set $m$ is a non-empty closed, convex subset of a non-empty convex set $M$ for which $x \in m, x=\lambda x_{1}+(1-\lambda) x_{2}$ with $0<\lambda<1$ and $x_{1} \in M, x_{2} \in M$ implies $x_{1} \in m, x_{2} \in m$.

The sets

$$
K_{0}(l):=\{V \mid V \in K, \mu(V, F)=0 \text { for all } F \in l \leqq \widehat{\hat{L}}\}
$$

are for all $l \leqq \hat{L}$ extremal set of $K$. Moreover, define for all $k \leqq K$

$$
\begin{array}{lll}
L_{0}(k):=\{F \mid F \in L, \mu(V, F)=0 & \text { for all } & V \in k \leqq K \\
\hat{L}_{0}(k):=\{F \mid F \in \hat{L}, \mu(V, F)=0 & \text { for all } & V \in k \leqq K\} \\
\hat{L}_{0}(k):=\{F \mid F \in \hat{L}, \mu(V, F)=0 & \text { for all } & V \in k \leqq K
\end{array} .
$$

The sets $\hat{L}_{0}(k)$ and $\widehat{L}_{0}(k)$ are extremal sets of $\hat{L}$ and $\widehat{L}$, respectively. The set $\hat{L}_{0}(k)$ is the closed convex hull of $L_{0}(k)$.

To axiom $2 \mathrm{a}$ it is equivalent that the sets $L_{0}(k)$ are ascending directed sets. Hence there follows that $L_{0}(k)$ has one and only one maximal element $E$. We call these $E$ 's decision effects. $G$ denotes the set of all $E$.

1 This is the axiom 2 in [1], p. 1314. There we have inadvertently forgotten the second part of the first principal law about measurement, stated here as the axiom $2 \mathrm{~b}$. 
If $k=\emptyset$, then $L_{0}(k)=L$. We denote the maximal element of $L$ by 1 . There holds $\mu(V, \mathbf{1})=1$ for all $V \in K$. We define the following sets

$$
\begin{array}{lll}
W=\left\{K_{0}(l) \mid l \leqq L\right\}, & \hat{W}=\left\{K_{0}(l) \mid l \leqq \hat{L}\right\}, & \hat{W}=\left\{K_{0}(l) \mid l \leqq \hat{L}\right\} \\
U=\left\{L_{0}(k) \mid k \leqq K\right\}, & \hat{U}=\left\{\hat{L}_{0}(k) \mid k \leqq K\right\}, & \hat{U}=\left\{\hat{L}_{0}(k) \mid k \leqq K\right\}
\end{array}
$$

By theorem $6, W=\hat{W}$. The sets $W, \hat{W}, \hat{W}, U, \hat{U}, \hat{U}$ are complete set lattices in which the lattice-theoretical intersection is equal to the settheoretical intersection, but the lattice-theoretical union can differ from the set-theoretical one.

By $K_{0}(l)$ with $l \in U, l \in \hat{U}$ and $l \in \hat{U}$, respectively, $K_{0}$ can be considered as a mapping of $U, \hat{U}$ and $\hat{U}$, respectively, onto $W, \hat{W}$ and $\hat{W}$, respectively. $K_{0}$ is then a dual isomorphism between the lattices $U$ and $W, \hat{U}$ and $\hat{W}$, $\widehat{U}$ and $\hat{W}$, respectively. The mappings $L_{0}, \hat{L}_{0}, \hat{L}_{0}$ are the reciprocal mappings of the mapping $K_{0}$ of $U$ onto $W$, of $\hat{U}$ onto $\hat{W}$ and of $\hat{U}$ onto $\hat{W}$, respectively. Whenever $l$ is a singleton $\{F\}$, we will use the shorthand notation $K_{0}(F)$ instead of $K_{0}(l)$. To each element $L_{0}(k)$ of $U$ a decision effect $E$ is attached bijectively and order preserving, which is the maximal element belonging to $L_{0}(k)$. Consequently $G$ is lattice-isomorphic with $U$. Thus $G$ is dual-isomorphic with $W=\hat{W}$, hence isomorphic with $\hat{U}$. It is even valid that $\hat{L}_{0}(k)$ and $L_{0}(k)$ have the same, only element $E$ being maximal. By $K_{0}, G$ is dual-isomorphically mapped onto $W=\hat{W}$.

\section{Principal Law About the Sensitivity-Increase of Effects (Second Part)}

Axiom $2 \mathrm{a}$ can be illustrated by filter packets $F_{1} F_{2} F_{1} F_{2} F_{1} F_{2} \ldots$ (as above briefly explained). For $F_{1}=F_{2}=F$ we obtain a filter packet FFF . . not identical with $F$. But axiom 2 a is trivially satisfied by $F_{3}=F_{1}=F_{2}$ if $F_{1}=F_{2}$. Therefore a possibility of increasing the sensitivity of an effect is not yet comprehended by axiom $2 \mathrm{a}$. A physically heuristic analysis more detailed (see [3]) shows that we have to express that there should exist for every $\lambda F$ with $\lambda \geqq 0, F \in \hat{L}$ and $\lambda \mu(V, F) \leqq 1$ another, more sensitive effect with the same $K_{0}(F)$. This possibility of sensitivity-increase can most simply be formulated by means of $\hat{\hat{L}}$.

For every $F \in \hat{L}$ we can infer from $K_{0}(F) \supseteqq K_{0}(E)$ with $E \in G$ that $F \leqq E$ holds. So, we can formulate the second part of the first principal law about measurement as follows:

Axiom 2 b. For any $F \in \hat{L}$ and any $E \in G$ with $K_{0}(F) \supseteqq K_{0}(E)$ there holds $F \leqq E$.

So, axiom $2 \mathrm{~b}$ is a postulated extension to $\hat{L}$ of a theorem valid for $\hat{L}$.

Theorem 1. If $F \in \hat{L}, \lambda \geqq 0$ and $\lambda \mu(V, F) \leqq 1$ for all $V \in K$, then $\lambda F \in \hat{L}$. 
Proof. If $F=\varrho F^{\prime}$ with $F^{\prime} \in \hat{L}$, then $\lambda F=\lambda \varrho F^{\prime} \in \hat{L}$ by definition of $\hat{L}$. If $F$ is the limit of elements of the form $\varrho F^{\prime}$ with $F^{\prime} \in \hat{L}$, then we may also choose such $F_{v} \in \hat{L}$, with $\sup \left\{\mu\left(V, F_{v}\right) \mid V \in K\right\}=1$ so that $\lambda_{v} F_{v} \rightarrow F$. Since $K$ is compact (in case $B$ finite-dimensional!), we have $\sup \left\{\mu\left(V, \lambda_{v} F_{v}\right) \mid V \in K\right\}=\lambda_{v} \rightarrow \sup \{\mu(V, F) \mid V \in K\}$. With $\eta$ : $=\sup \{\mu(V, F) \mid V \in K\}$ we have $\eta F_{v} \rightarrow F$, too; thus $\lambda \eta F_{v} \rightarrow \lambda F$ and hence, because of $\sup \left\{\mu\left(V, \lambda \eta F_{\nu}\right) \mid V \in K\right\}=\lambda \eta \leqq 1, \lambda F \in \hat{L}$ finally.

Let $\mathscr{P}$ denote the cone $\left\{Y \mid Y \in B^{\prime}, Y \geqq 0\right\}$ and let $\mathscr{Q}$ denote the cone $\{X \mid X \in B, \mu(X, Y) \geqq 0$ for all $Y \in \mathscr{P}\}$. By the bipolar theorem, e.g. [6], p. 94, $\mathscr{Q}$ is then the closed, convex hull of $K \vee\{0\}$, i.e. the closed cone generated by $K$.

Theorem 2. $\mathscr{Q}=\{X \mid X=\lambda V, \lambda \geqq 0, V \in K\}$.

Proof. We have only to prove that $\lambda_{v} \cdot V_{v} \rightarrow X$ also implies $X=\lambda V$ with suitable $\lambda \geqq 0$ and $V \in K$.

From $\lambda_{v} \cdot V_{v} \rightarrow X$ there results $\lambda_{v} \rightarrow \mu(X, 1)$. To exclude triviality, remark that, because of $\mu\left(V_{p}, F\right) \leqq 1$ for all $F \in L, \mu(X, 1)=0$ also implies $\mu(X, F)=0$ for all $F \in L$, hence $X=0 \in \mathscr{Q}$.

If $\mu(X, \mathbf{1}) \neq 0$, then $V_{v} \rightarrow X \cdot \mu(X, \mathbf{1})^{-1}$, i.e. the sequence $\left(V_{v}\right)$ converges and, since $K$ is closed, $V_{v} \rightarrow V \in K$; thus $X=\mu(X, 1) \cdot V \in \mathscr{Q}$.

Theorem 3. The cone

$\mathscr{P}_{+}=\left\{Y \mid Y \in B^{\prime}, \mu(V, Y) \leqq 1\right.$ for all $\left.V \in K\right\}$ equals the set $1-\mathscr{P}:=\{1-Y \mid Y \in \mathscr{P}\}$.

Proof. $\mu(V, Y) \leqq 1$ implies $\mu(V, 1-Y) \geqq 0$ and vice versa.

Theorem 4. The closed, convex cone generated by, $\hat{L}$ and $\hat{L}$, respectively is equal to

$$
\underset{\lambda \geqq 0}{\bigvee} \lambda \hat{L}=\{Y \mid Y=\lambda F, \lambda \geqq 0, F \in \widehat{\hat{L}}\}^{2} .
$$

Proof. It is immediately evident that $L, \hat{L}$ and $\hat{L}$, respectively, generate the same closed, convex cone. It only remains to show that $\lambda_{\nu} \cdot F_{\nu} \rightarrow Y$ with $F_{\nu} \in \hat{\hat{L}}, \lambda_{\nu} \geqq 0$ implies $Y=\lambda F$ with suitable $\lambda \geqq 0$ and $F \in \hat{L}$. We may choose $\left(F_{v}\right)$ so that $\sup \left\{\mu\left(V, F_{v}\right) \mid V \in K\right\}=1$. As in the proof of theorem 1 there follows $\lambda_{v} \rightarrow \sup \{\mu(V, Y) \mid V \in K\}$.

$Y=0$ is trivial. If $Y \neq 0$, there results that the sequence $\left(F_{v}\right)$ converges, i.e. $F_{v} \rightarrow F \in \hat{\hat{L}}$; thus $Y=F \sup \{\mu(V, Y) \mid V \in K\}$.

An immediate conquence of theorem 4 is the

Theorem 5. $\hat{L}=\underset{\lambda \geq 0}{\mathrm{~V}} \lambda \hat{\bar{L}} \wedge \mathscr{P}_{+}$.

\section{Principal Law About Decomposability and Relationship of Effects}

For each $V \in K$ there exists the extremal set generated by $V$, which we denote by $C(V)$. Thus $C(V)$ is the intersection of all extremal sets of $K$ containing $V$. Every extremal set of $K$ can be written as $C(V)$ with

${ }^{2}$ In this paper $\Lambda, V$ denote the set-theoretical intersection and union, respectively; whereas $\cap, \cup$ denote the lattice-theoretical intersection and union, respectively. 
$V$ suitable. For we need only choose an internal point of the extremal set, which exists because $B$ is finite-dimensional. $B$ finite-dimensional, $C(V)$ is the set $\left\{V^{\prime} \mid V^{\prime} \in K, V=\lambda V^{\prime}+(1-\lambda) V^{\prime \prime}\right.$ with $0<\lambda<1$ and $\left.V^{\prime \prime} \in K\right\}$ i.e. the set of all possible mixture components of $V$. As in [1] and [3] physically explained in detail, we postulate as the second principal law about measurement:

Axiom 3. $L_{0}\left(V_{1}\right)=L_{0}\left(V_{2}\right)$ implies $C\left(V_{1}\right)=C\left(V_{2}\right)$ for all $V_{1}, V_{2} \in K$. $L_{0}(V)=L_{0}(C(V))$ always holding, we infer from axiom 3 that $L_{0}\left(V_{1}\right)=L_{0}\left(V_{2}\right)$ is equivalent to equivalent to $C\left(V_{1}\right)=C\left(V_{2}\right)$ for all $V_{1}, V_{2} \in K$.

Theorem 6. i) $C(V)=K_{0} L_{0}(V)$ for each $V \in K$,

ii) $W$ contains all extremal sets of $K$,

iii) $\hat{W}=\hat{W}=W$ and $K_{0} \hat{L}_{0}(V)=K_{0} \hat{L}_{0}(V)=K_{0} L_{0}(V)$ for each $V \in K$.

Proof. i) Assuming $K_{0} L_{0}(V) \neq C(V)$, then we get $L_{0} K_{0}(V) \neq L_{0}(C(V))$ by axiom 3. Because of $L_{0} K_{0} L_{0}(V)=L_{0}(V)=L_{0}(C(V))$ this is a contradiction.

ii) An immediate consequence of i).

iii) Since $\hat{W} \supseteqq \hat{W} \supseteqq W$ and $\hat{W}$ consists of extremal sets of $K$, ii) implies $\widehat{W}=W$. From $\widehat{L}_{0}(V) \supseteqq \hat{L}_{0}(V) \supseteqq L_{0}(V)$ we infer $K_{0} \widehat{\hat{L}}_{0}(V) \leqq K_{0} \hat{L}_{0}(V)$ $\leqq K_{0} L_{0}(V)=C(V)$.

By definition of $C(V), K_{0} \widehat{\hat{L}}_{0}(V) \supseteqq C(V)$, thus $K_{0} \widehat{\hat{L}}_{0}(V)=K_{0} \hat{L}_{0}(V)$ $=K_{0} L_{0}(V)$.

Theorem 7. For every $k \leqq K, \widehat{\widehat{L}}_{0}(k)$ contains one and only one maximal element $E$, which is the maximal element of $L_{0}(k)$.

Proof. Because of $\widehat{\hat{L}}_{0}(k) \supseteqq L_{0}(k)$ there holds $E \in \widehat{\hat{L}}_{0}(k), E$ being the maximal element of $L_{0}(k)$. Suppose $F \in \widehat{\hat{L}}_{0}(k)$, hence $K_{0}(F) \supseteqq K_{0} \widehat{\hat{L}}_{0}(k)$ $=K_{0} L_{0}(k)=K_{0}(E)$ by theorem 6 . Then axiom $2 \mathrm{~b}$ implies $F \leqq E$.

Theorem 8. i) $\underset{\lambda \geqq 0}{\mathrm{~V}} \lambda \widehat{\mathrm{L}}=\mathscr{P}$.

ii) $\widehat{\hat{L}}=\mathscr{P} \wedge \mathscr{P}_{+}$, i.e. $\hat{\hat{L}}=\left\{Y \mid Y \in B^{\prime}, \quad 0 \leqq \mu(V, Y) \leqq 1\right.$ for all $V \in K\}$.

iii) $K=\{X \mid X \in B, 0 \leqq \mu(X, F)$ for all $F \in \hat{\hat{L}}$ and $\mu(X, 1)=1\}$.

Proof. Since obviously $\underset{\lambda \geq 0}{\bigvee} \lambda \hat{L} \leqq \mathscr{P}$, there holds for the corresponding polar sets $\mathscr{P} 0 \leqq\left(\mathrm{~V}_{\lambda \geqq 0} \lambda \hat{L}\right)^{0}$. Thus the proof will be achieved by showing $(\underset{\lambda \geqq 0}{\mathrm{~V}} \lambda \hat{\bar{L}})^{0} \leqq \mathscr{P} 0$. For then there holds for the bipolar sets $(\underset{\lambda \geqq 0}{\mathrm{~V}} \lambda \widehat{\hat{L}})^{00}=\mathscr{P} 00$ and, since $\underset{\lambda \geqq 0}{\mathrm{~V}} \lambda \widehat{\hat{L}}$ and $\mathscr{P}$ are closed, convex sets, $\lambda_{\lambda \geq 0}^{\mathrm{V}} \lambda \hat{\hat{L}}=\mathscr{P}$ is valid according to the bipolar theorem. To verify $(\underset{\lambda \geq 0}{\bigvee} \lambda \hat{\bar{L}})^{0} \leqq \mathscr{P} 0$, it suffices to prove that for every $X \notin \mathscr{Q}$ there exists $F \in \hat{L}$ with $\mu(X, F)<0$. Select an internal point $V$ of $K$, which exists because $B$ is finite-dimensional. Then $C(V)=K$ is valid. Suppose $X \notin \mathscr{Q}$, hence the line through 
$V$ and $X$ meets the boundary of the cone $\mathscr{Q}$ in a point $X^{\prime}$. If $X^{\prime}=0$, then $\mu(X, \mathbf{1})<0$ because of $\mu(V, \mathbf{1})=1$. If $X^{\prime} \neq 0$, i.e. $X^{\prime}=\lambda V^{\prime}$ with $\lambda>0$ and $V^{\prime}$ on the boundary of $K$, we have $C\left(V^{\prime}\right) \neq C(V)=K$ because $V^{\prime}$ cannot have $V$ as a mixture component. $C\left(V^{\prime}\right) \neq K$ implies $L_{0}\left(V^{\prime}\right)$ $\neq L_{0}(V)=\{0\}$. Thus there exists $F \in L$ (hence $\left.F \in \hat{L}\right)$ so that $\mu(V, F)>0$, $\mu\left(V^{\prime}, F\right)=0$ and hence $\mu(X, F)<0$.

\section{The Structure of the Lattice $G$ of the Decision Effects}

Because of the importance of the following theorems let us here repeat some theorems of [5] with proofs partially simplified.

Theorem 9. Every $F \in \hat{\hat{L}}$ admits a unique decomposition of the form

$$
\begin{gathered}
F=\sum_{\nu=1}^{n} \lambda_{\nu}\left(E_{v}-E_{v+1}\right), \text { where } E_{v} \in G, E_{v+1}<E_{v}, \\
E_{n+1}=0,0<\lambda_{\nu}<1 \text { for all } v>1,0<\lambda_{1} \leqq 1
\end{gathered}
$$

and $\lambda_{\nu} \neq \lambda_{\mu}$ for $v \neq \mu$.

Proof. We define $E_{1}$ by $K_{0}(F)=K_{0}\left(E_{1}\right)$ (thus $E_{1}$ is the maximal element of $\left.\hat{\bar{L}}_{0} K_{0}\left(E_{1}\right) !\right)$. As a consequence, $F \leqq E_{1}$. Define $\alpha_{1}=\sup \{\mu(V, F) \mid V \in K\}$, then $\alpha^{-1} F \leqq E_{1}$ too.

Therefore we have $F_{1}=E_{1}-\alpha^{-1} F \in \hat{L}$. By $K_{0}\left(F_{1}\right)=K_{0}\left(E_{2}\right)$ we define $E_{2}$. From $F_{1} \leqq E_{1}$ there follows at once $E_{2} \leqq E_{1}$. There even holds $E_{2} \neq E_{1}$ : since $K$ is compact, $\mu\left(\cdot, \alpha_{1}^{-1} F\right)$ attains its supremum on $K$, there holds for such a $V_{0} \mu\left(V_{0}, \alpha_{1}^{-1} F\right)=1$, thus $\mu\left(V_{0}, E_{1}\right)=1$ but $\mu\left(V_{0}, F_{1}\right)=0$, hence $K_{0}\left(F_{1}\right) \neq K_{0}\left(E_{1}\right)$ which implies the assertion. Moreover, $\alpha_{2}=\sup \left\{\mu\left(V, F_{1}\right) \mid V \in K\right\}<1$ is valid: for assume the existence of $V^{\prime}$ with $1=\mu\left(V^{\prime}, F_{1}\right)=\mu\left(V^{\prime}, E_{1}-\alpha_{1}^{-1} F\right)=\mu\left(V^{\prime}, E_{1}\right)$ $-\alpha_{1}^{-1} \cdot \mu\left(V^{\prime}, F^{\prime}\right)$. This implies $\mu\left(V^{\prime}, E_{1}\right)=1$ and $\mu\left(V^{\prime}, F^{\prime}\right)=0$, hence $K_{0}(F) \neq K_{0}\left(E_{1}\right)$ which is a contradiction. If $\alpha_{2} \neq 0$, then we can define $F_{2}=E_{2}-\alpha_{2}^{-1} F_{1} \in \hat{L}$ and extend the sequences $\left(F_{v}\right)$ and $\left(E_{v}\right)$ by recurrence until $\alpha_{n+1}$ vanishes, whence $F_{n}=E_{n+1}=0$. Such finite integer $n$ exists for, because of the finite dimension of $B^{\prime}$ and the increasing dimension of $K_{0}\left(E_{v}\right)$, the sequence $\left(E_{v}\right)$ must break off after finitely many steps. So we obtain the finitely many equations:

$$
\begin{gathered}
\alpha_{1} F_{1}=\alpha_{1} E_{1}-F \\
\alpha_{2} F_{2} \alpha_{2} E_{2}-F_{1} \\
\vdots \\
\alpha_{n-1} F_{n-1}=\alpha_{n-1} E_{n-1}-F_{n-1} \\
0=\alpha_{n} E_{n}-F_{n-1}^{\prime}
\end{gathered}
$$


where $0<\alpha_{v}<1$ for $v>1,0<\alpha_{1} \leqq 1$ and $E_{v+1}<E_{v}$. From these finitely many equations there follows

$$
F=\sum_{\nu=1}^{n}(-1)^{\nu+1} \beta_{\nu} E_{\nu} \quad \text { with } \quad \beta_{\nu}=\prod_{\varrho=1}^{\nu} \alpha_{\varrho} .
$$

Using the identity $E_{v}=\sum_{\varrho=v}^{n}\left(E_{\varrho}-E_{\varrho+1}\right)$ with $E_{n+1}=0$, we can write $F^{\prime}=\sum_{\nu=1}^{n} \lambda_{v}\left(E_{v}-E_{v+1}\right)$ with $\lambda_{\nu}=\sum_{\varrho=1}^{\nu}(-1)^{\varrho+1} \beta_{\varrho}=\sum_{\varrho=1}^{v}(-1)^{\varrho+1} \prod_{\sigma=0}^{\varrho} \alpha_{\sigma}$. Since $0<\alpha_{v}<1$ for $\nu>1$ and $0<\alpha_{1} \leqq 1$, so $0<\lambda_{v}<1$ for $\nu>1$ and $0<\lambda_{1} \leqq 1$ Uniqueness results from the fact that the $E_{v}$ 's in the above decomposition are unique because they are maximal elements (and thus unique). The $\lambda_{v}$ 's are also unique because the $\alpha_{v}$ 's are suprema, hence unique. This completes the proof.

Theorem 10. The set of all extreme points of $\hat{L}$ is equal to $G$.

Proof. 1) Every $E \in G$ is an extreme point of $\hat{L}$ : for $E=\lambda F_{1}+(1-\lambda) F_{2}$ with $F_{1}, F_{2} \in \hat{\bar{L}}$ and $0<\lambda<1$ implies $K_{0}(E) \leqq K_{0}\left(F_{1}\right) \cap K_{0}\left(F_{2}\right)$, hence $F_{1} \leqq E$ and $F_{2} \leqq E$.

Assume $F_{1}<E$, then there exists $V \in K$ with $\mu\left(V, F_{1}\right)<\mu(V, E)$; thus $\mu(V, E)=\lambda \mu\left(V, F_{1}\right)+(1-\lambda) \mu\left(V, F_{2}\right)<\lambda \mu(V, E)+(1-\lambda) \mu(V, E)$ $=\mu(V, E)$, which is a contradiction. So, $F_{1}=F_{2}=E$ is valid.

2) If $F \in \hat{\hat{L}}$ but $F \notin G$, then according to theorem $9 F=\sum_{\nu=1}^{n} \lambda_{\nu}\left(E_{\nu}-E_{\nu+1}\right)$, where at least one $\lambda_{v}$, say $\lambda_{\varrho}$, satisfies $0<\lambda_{\varrho}<1$. Choose $\varepsilon>0$ so that $\lambda_{\varrho}-\varepsilon>0$ and $\lambda_{\varrho}+\varepsilon<1$.

Put $F_{ \pm}=\sum_{\nu \neq \varrho}^{n} \lambda_{v}\left(E_{v}-E_{v+1}\right)+\left(\lambda_{\varrho} \pm \varepsilon\right)\left(E_{\varrho}-E_{\varrho+1}\right)$, then there holds $0 \leqq F_{ \pm} \leqq \sum_{\nu=1}^{n}\left(E_{\nu}-E_{\nu+1}\right)=E_{1} \leqq 1$, thus $F_{ \pm} \in \hat{\bar{L}}$ by theorem $\left.8 \mathrm{ii}\right)$. Moreover, we can infer $F=\frac{1}{2} F_{+}+\frac{1}{2} F_{-}$, thus $F$ is not an extreme point of $\hat{\bar{L}}$.

Theorem 11. $\hat{L}=\widehat{\hat{L}}$.

Proof. The convex set $\hat{L}$ contains $G$, which is the set of all extreme points of $\hat{L}$ according to the preceding theorem. Therefore we conclude, by the theorem of KREIN-MILMAN, $\hat{L}=\hat{L}$.

Theorem 12. For all elements $E_{1}, E_{2} \in G$ with $E_{1} \leqq E_{2}$ holds $E_{2}-E_{1} \in G$.

Proof. Because of $E_{1} \leqq E_{2}$ we have $0 \leqq E_{2}-E_{1} \leqq E_{2} \leqq 1$, hence $E_{2}-E_{1} \in \hat{\hat{L}}=\hat{L}$. We shall prove that $E_{2}-E_{1}$ is an extreme point of $\hat{\bar{L}}$ : assume that $E_{2}-E_{1}=\lambda F_{1}+(1-\lambda) F_{2}$ with $0<\lambda<1$ and $F_{1}, F_{2} \in \hat{\hat{L}}$. Hence there follows because of $E_{2}-E_{1} \leqq E_{2} K_{0}\left(F_{1}\right) \wedge K_{0}\left(F_{2}\right) \supseteqq K_{0}\left(E_{2}\right)$, 
thus $F_{1} \leqq E_{2}$ and $F_{2} \leqq E_{2}$. So we can write $E_{1}=E_{2}-\left(E_{2}-E_{1}\right)$ $=\lambda\left(E_{2}-F_{1}\right)+(1-\lambda)\left(E_{2}-F_{2}\right) . E_{1}$ being an extreme point, we infer $E_{2}-F_{1}=E_{1}, E_{2}-F_{2}=E_{1}$, i.e. $F_{1}=F_{2}=E_{2}-E_{1}$, which implies that $E_{2}-E_{1}$ is an extreme point of $\hat{\hat{L}}$, thus by theorem $10, E_{2}-E_{1} \in G$. So, $E_{2}-E_{1}$ is a decision effect.

Theorem 13. The mapping $*: G \rightarrow G$ defined by $E \rightarrow E^{*}=\mathbf{1}-E$ for all $E \in G$ is an orthocomplementation of the lattice $G$.

Proof. According to theorem 12 we have at once $E^{*} \in G$ for all $E \in G$. It is immediately evident that the mapping $E \rightarrow E^{*}$ is a dual automorphism of $G .1-E^{*}=E$ implies $\left(E^{*}\right)^{*}=E . E+E^{*}=1$ implies $K_{0}(E) \wedge K_{0}\left(E^{*}\right)=\emptyset$, i.e. $E \cup E^{*}=1$, hence $E^{*} \cap E=1^{*}=0$. As it well-known, two elements $E_{1}, E_{2} \in G$ are called orthogonal (symbolically $E_{1} \perp E_{2}$ ) if $E_{1} \leqq E_{2}^{*}$. This relation is obviously symmetrical. So $E *$ is the orthocomplement of $E$ with respect to the mapping $*$. This completes the proof.

By theorem 12, $E_{1} \perp E_{2}$ implies $E_{2}^{*}-E_{1} \in G$, thus $1-\left(E_{2}^{*}-E_{1}\right)$ $=E_{2}+E_{1} \in G$. Hence we obtain $K_{0}\left(E_{1}\right) \wedge K_{0}\left(E_{2}\right)=K_{0}\left(E_{1}+E_{2}\right)$ and thus, because of $K_{0}\left(E_{1}\right) \wedge K_{0}\left(E_{2}\right)=K_{0}\left(E_{1} \cup E_{2}\right), E_{1}+E_{2}=E_{1} \cup E_{2}$ finally. Given a sequence of pairwise orthogonal $E_{v}(v=1, \ldots, n)$, then $E_{v+1} \perp E_{\varkappa} \quad(\varkappa \leqq v)$ implies $E_{v+1} \leqq E_{\varkappa}^{*}$ and thus $E_{v+1}$ $\leqq \bigcap_{x=1}^{\nu} E_{\varkappa}^{*}=\left(\bigcup_{x=1}^{\nu} E_{\varkappa}\right)^{*}$.

Hence we can conclude $E_{\nu+1} \perp \sum_{x=1}^{\nu} E_{x}$ by assuming $\bigcup_{x=1}^{\nu} E_{\varkappa}=\sum_{x=1}^{\nu} E_{x}$ to be valid. Thus there hold $E_{\nu+1}+\sum_{\varkappa=1}^{\nu} E_{\varkappa} \in G$ and $E_{\nu+1}+\sum_{\varkappa=1}^{\nu} E_{\varkappa}$ $=\sum_{\varkappa=1}^{v+1} E_{\varkappa}=E_{v+1} \cup \sum_{\varkappa=1}^{v} E_{\varkappa}=E_{v+1} \cup\left(\bigcup_{\varkappa=1}^{v} E_{\varkappa}\right)=\bigcup_{\varkappa=1}^{v+1} E_{\varkappa}$.

So we have, by mathematical induction, proved the following theorem:

Theorem 14. Any finite sequence $\left(E_{v}\right)(\nu=1, \ldots, n)$ of pairwise orthogonal $E_{v} \in G$ satisfies

$$
\sum_{\nu=1}^{n} E_{\nu}=\bigcup_{\nu=1}^{n} E_{\nu}
$$

Theorem 14 expresses the important fact of the orthoadditivity of the measures $\mu(V, \cdot)$ on $G$. When $B$ is finite-dimensional, only finitely many, pairwise orthogonal $E_{\nu} \in G$ can exist.

Theorem 15. For every $F \in \hat{L}=\hat{L}$ there holds $F=\sum_{x=1}^{n} \lambda_{x} \cdot E_{\varkappa}$ with pairwise orthogonal $E_{\varkappa} \in G$ and $1 \geqq \lambda_{1}>\lambda_{2}>\cdots>\lambda_{n}>0$.

Proof. It is an immediate consequence of the theorems 9 and 12. For the elements $E_{v}-E_{v+1}$ of theorem 9 are pairwise orthogonal: $E_{v}>E_{v+1}$ implies $E_{\nu}-E_{v+1} \leqq 1-E_{v+1}=E_{v+1}^{*}$, hence we have for $v<\varkappa$, because of $E_{v}>E_{\varkappa}, E_{\nu}-E_{v+1} \leqq E_{v+1}^{*} \leqq E_{\varkappa}^{*} \leqq\left(E_{\varkappa}-E_{\varkappa+1}\right)^{*}$. Finally we can antitonely rearrange the $\lambda_{\nu}$ 's of theorem 9 . 
Theorem 14 implies:

Theorem 16. $G$ is orthomodular.

Proof. Call a lattice orthomodular if $E_{2} \perp E_{1}, E_{2}^{\prime} \perp E_{1}$ and $E_{1} \cup E_{2}$ $=E_{1} \cup E_{2}^{\prime}$ implies $E_{2}=E_{2}^{\prime}$ (e.g. [7]). The lattice $G$ satisfies this condition: by theorem 14 there holds $E_{1} \cup E_{2}=E_{1}+E_{2}$ and $E_{1} \cup E_{2}^{\prime}=E_{1}+E_{2}^{\prime}$. Thus $E_{1}+E_{2}=E_{1}+E_{2}^{\prime}$ implies $E_{2}=E_{2}^{\prime}$ at once.

Let $E_{1}, E_{2}, E_{3}$ be three elements of $G$ with $E_{1} \geqq E_{2}, E_{2} \perp E_{3}$. Then the modular relation is valid (e.g. [7]):

$$
E_{1} \cap\left(E_{2} \cup E_{3}\right)=E_{2} \cup\left(E_{1} \cap E_{3}\right) .
$$

If we could drop the subsidiary condition $E_{2} \perp E_{3}$, the lattice would be modular.

\section{Principal Law About the Components of the Mixture of Two Ensembles}

As the third principal law about measurement we formulate (in case of a finite-dimensional $B$ ):

Axiom 4. For all $V_{1}, V_{2}, V_{3} \in K$ :

$C\left(V_{1}\right) \wedge C\left(V_{2}\right)=\emptyset$ and $\emptyset \neq C\left(V_{3}\right) \leqq C\left(\frac{1}{2} V_{1}+\frac{1}{2} V_{2}\right)$ and $d\left(V_{1}, V_{3}\right)=1$ implies $C\left(\frac{1}{2} V_{1}+\frac{1}{2} V_{3}\right) \wedge C\left(V_{2}\right) \neq \emptyset$.

$d\left(V_{1}, V_{2}\right)$ is defined by $d\left(V_{1}, V_{2}\right)=\sup \left\{\left|\mu\left(V_{1}, F\right)-\mu\left(V_{2}, F\right)\right| \mid F \in \hat{L}\right\}$.

A physical interpretation of this axiom was given in [2] and [3]. Let us briefly show that modularity of $G$ follows from axiom 4 . It is sufficient to prove the modularity of the lattice $W$ consisting of the extremal sets $C(V)$ of $K$, which is dual-isomorphic with $G$.

Theorem 17. $G$ and $W$ are modular.

Proof. Because of the order isomorphism between $G$ and $W$ given by $E \leftrightarrow K_{0}\left(E^{*}\right)$, it suffices to verify the theorem for $W$. From $d\left(V_{1}, V_{2}\right)=1$ there results the existence of at least one $F \in \hat{L}$ so that $\mu\left(V_{1}, F\right)=1$ and $\mu\left(V_{2}, F\right)=0$ (if $\mu\left(V_{1}, F^{\prime}\right)=0$ and $\mu\left(V_{2}, F^{\prime}\right)=1$, then $F=\mathbf{1}-F^{\prime}$ satisfies $\mu\left(V_{1}, F\right)=1$ and $\mu\left(V_{2}, F\right)=0$ !). Thus $V_{1} \in K_{0}(1-F)$ and $V_{2} \in K_{0}(F)$. With $K_{0}(F)=K_{0}(E)$ there consequently holds $V_{1} \in K_{0}\left(E^{*}\right)$, $V_{2} \in K_{0}(E)$ and hence $C\left(V_{1}\right) \subseteq K_{0}\left(E^{*}\right), C\left(V_{2}\right) \leqq K_{0}(E)$. By the validity of $C\left(V_{1}\right)=K_{0}\left(E_{1}^{*}\right)$ and $C\left(V_{2}\right)=K_{0}\left(E_{2}\right)$, the decision effects $E_{1}$ and $E_{2}$ are isomorphically attached to $C\left(V_{1}\right)$ and $C\left(V_{2}\right)$, respectively. Thus $E_{1}^{*} \geqq E^{*}$ and $E_{2}^{*} \geqq E$, i.e. $E_{1} \leqq E \leqq E_{2}^{*}$, hence $E_{1} \perp E_{2}$.

Conversely, if $E_{1} \perp E_{2}$ (in this case we briefly write $C\left(V_{1}\right) \perp C\left(V_{2}\right)$ ), then we have $E_{2} \leqq E_{1}^{*}$ and, because of $C\left(V_{1}\right)=K_{0}\left(E_{1}^{*}\right) \leqq K_{0}\left(E_{2}\right)$ and $C\left(V_{2}\right)=K_{0}\left(E_{2}^{*}\right)$, we obtain $\mu\left(V_{2}, E_{2}^{*}\right)=0$ and $\mu\left(V_{1}, E_{2}\right)=0$. Thus $d\left(V_{1}, V_{2}\right)=1$.

Let us introduce the abbreviations $a=C\left(V_{1}\right), \quad b=C\left(V_{2}\right)$ and $c=C\left(V_{3}\right) . \quad V_{3} \in C\left(\frac{1}{2} V_{1}+\frac{1}{2} V_{2}\right)$ (one of the conditions of axiom 4) implies $C\left(V_{3}\right) \leqq C\left(\frac{1}{2} V_{1}+\frac{1}{2} V_{2}\right)$. Since $C\left(\frac{1}{2} V_{1}+\frac{1}{2} V_{2}\right)=C\left(V_{1}\right) \cup C\left(V_{2}\right)$ 
$=a \cup b, c \leqq a \cup b$ is finally valid. Likewise, we have $C\left(\frac{1}{2} V_{1}+\frac{1}{2} V_{3}\right)$ $=a \cup c$. As we have seen, $d\left(V_{1}, V_{3}\right)=1$ is equivalent with $a \perp c$. So, axiom 4 can be written in the form:

Axiom $4^{\prime} \cdot a \cap b=0, c \leqq a \cup b, a \perp c$ and $(a \cup c) \cap b=0$ implies $c=0$.

To continue the proof of theorem 17, let us consider three elements $d, e, f$ of $W$ with $d \geqq e . W$ being orthomodular, there exists $b \perp(d \cap f)$ so that $f=(d \cap f) \cup b$. Therefore $d \cap[(d \cap f) \cup b] \geqq d \cap(d \cap f)=d \cap f$ and $d \cap[(d \cap f) \cup b] \geqq d \cap b$, hence $d \cap f=d \cap[(d \cap f) \cup b]$ $\geqq(d \cap f) \cup(d \cup b)$. Since $d \cap b \perp d \cap f$, orthomodularity yields $d \cap b$ $=(d \cap f)^{*} \cap[(d \cap f) \cup(d \cap b)]$, thus $d \cap b=0$. Putting $a=e \cup(d \cap f)$, we obtain

$$
d \cap(e \cup f)=d \cap[e \cup(d \cap f) \cup b]=d \cap(a \cup b),
$$

where $d \cap b=0$ and $d \geqq a$ because of $d \geqq e$ and $d \geqq d \cap f$. Abbreviating $d \cap(a \cup b)$ by $l$, we have $a \cup b \geqq l, l \cap b=d \cap(a \cup b) \cap b$ $=d \cap b=0$ and $l \geqq d \cap a=a$. Therefore there exists $c$ with $c \perp a$ and $l=a \cup c . c \leqq l$ implies $c \leqq a \cup b$. Besides, there holds $0=l \cap b$ $=(a \cup c) \cap b . a \leqq l$ implies $a \cap b \leqq l \cap b=0$. Thus axiom $4^{\prime}$ applies and so $c=0$, hence $l=a$, i.e. $d \cap(a \cup b)=a$. Then (1) has the form $d \cap(e \cup f)=a=e \cup(d \cap f)$, which is the modular relation between $d, e, f$.

\section{The Atoms of the Lattice of the Decision Effects}

By the mapping $E \leftrightarrow K_{0}\left(E^{*}\right)=C(V)$ already repeatedly used above, $G$ is lattice-isomorphically mapped onto the lattice $W$ of the extremal sets of $K$. Because of the finite dimension of $B, G$ must, therefore, be atomic. When $P$ is an atom of $G$ then $C(V)=K_{0}\left(P^{*}\right)$ must be an atom of $W$, i.e. an extreme point of $K: C(V)=\{V\}$. So, to each atom $P \in G$ the extreme point $V \in K$ is bijectively attached satisfying $\mu(V, P)=1$. Thus the extreme points of $K$ correspond biunivocally to the atoms of $G$, whereas the extreme points of $\hat{L}$ form the whole of $G$.

The finite dimension of $B$ implies the validity of the finite chain condition: an isotone sequence $\left(E_{v}\right)$, i.e. $E_{v+1}>E_{v}$, has only finitely many links. Since $G$ is modular, all maximal chains between two elements $E_{0}, E$ with $E_{0}>E$ have the same length (e.g. [8]). Remember that a maximal chain is a sequence $\left(E_{v}\right)(v=1, \ldots, n)$ with $E_{v+1}>E_{v}$ and $E_{n}=E$, where no $E^{\prime}$ can be interpolated between any two links $E_{v}<E_{v+1}$ so that $E_{v}<E^{\prime}<E_{v+1}$ holds. From this it follows especially for two sequences $\left(P_{v}\right)_{v \in N},\left(P_{v}^{\prime}\right)_{v \in N^{\prime}}$ with $P_{v_{0}} \cap\left(\underset{\substack{v \in N \\ v \neq v_{0}}}{\bigcup_{n}} P_{v}\right)=0$ and $P_{v_{0}}^{\prime} \cap\left(\bigcup_{\substack{\nu \in N^{\prime} \\ v \neq v_{0}}} P_{v}^{\prime}\right)$ that $\bigcup_{v=1}^{n} P_{v}=\bigcup_{\nu=1 \ldots n^{\prime}} P_{\nu}^{\prime}$ implies $n=n^{\prime}$. In particular, if the $P_{\nu}^{\prime}$ 's are pairwise orthogonal then $P_{v_{0}} \cap\left(\bigcup_{\substack{v \in N \\ v \neq v_{0}}} P_{v}\right)=0$ is valid. Thus the maximal number of pairwise orthogonal atoms $P_{\nu} \leqq E$ depends only on $E$. We shall use this fact in the proofs of the following two theorems. 
Theorem 18. The set of all atoms of $G$ is closed.

Proof. It must be shown that any sequence $\left(P_{v}\right)$ of atoms of $G$ convergent in the space $B^{\prime}$ converges to an atom $P$ of $G: P_{v} \rightarrow P$. By $K_{0}\left(P_{v}^{*}\right)=C\left(V_{v}\right)=\left\{V_{v}\right\}$ the extreme point $V_{v}$ is biunivocally attached to $P_{v} . K$ being compact, we can choose a subsequence so that $\left(V_{v}\right)$ also converges. Thus we may suppose $V_{\nu} \rightarrow V$. $G$ being modular, the cardinal number of a "complete" system of pairwise orthogonal atoms $Q_{i}$ is independent of the system. That is for any two of such complete systems, i.e. $\sum_{i=1}^{n} Q_{i}=\mathbf{1}=\sum_{i=1}^{n^{\prime}} Q_{i}^{\prime}, n=n^{\prime}$ is valid.

Each $P_{v}$, can be so supplemented as to belong to a complete system of orthogonal atoms $Q_{\nu}^{(i)}$ with $Q_{\nu}^{(1)}=P_{v}$ and $\sum_{i=1}^{n} Q^{i)}=\mathbf{1}$. In case of need, we can manage by selecting subsequences that for all $Q_{\nu}^{(i)}: Q_{\nu}^{(i)} \rightarrow Q^{(i)} \in \hat{L}$ and for the $V_{\nu}^{(i)}$ pertaining: $V_{\nu}^{(i)} \rightarrow V^{(i)} \in K . \sum_{i=1}^{n} Q_{\nu}^{(i)}=\mathbf{1}$ also implies $\sum_{i=1}^{n} Q^{(i)}=1$. According to theorem $15, Q^{(i)}=\sum_{\varrho} \lambda_{\varrho}^{(i)} \cdot E_{0}^{(i)},\left(\lambda_{\varrho}^{(i)}\right)$ antitone with respect to $\varrho$. Since $\mu\left(V_{v}^{(i)}, Q_{\nu}^{(i)}\right)=1$, so for the limit $\mu\left(V^{(i)}, Q^{(i)}\right)=1$ too. Thus, by theorem $9, \lambda_{1}^{(i)}=1$. Assume $Q^{(i)}$ not to be an atom. Then it can be written as $Q^{(i)}=\bar{Q}^{(i)}+R^{(i)}$ where $\bar{Q}^{(i)}$ is an atom and $R^{(i)} \neq 0$. Hence $\mathbf{1}=\sum_{i=1}^{n} Q^{(i)}=\sum_{i=1}^{n} \bar{Q}^{(i)}+\sum_{i=1}^{n} R^{(i)} \cdot \sum_{i=1}^{n} \bar{Q}^{(i)} \leqq \mathbf{1}$ implies $\sum_{i \neq j} \bar{Q}^{(i)}$ $\leqq 1-\bar{Q}^{(j)}=\bar{Q}^{(j) *}$, thus $\bar{Q}^{(i)} \leqq \bar{Q}^{(j) *}$ for $i \neq j$ and so $\bar{Q}^{(i)} \perp \bar{Q}^{(j)}$. This orthogonality implies $\sum_{i=1}^{n} \bar{Q}^{(i)}=1$, for, otherwise, there would exist a system of more than $n$ pairwise orthogonal atoms. Thus there holds $\sum_{i=1}^{n} R^{(i)}=0$, and from $R^{(i)} \geqq 0$ we infer $R^{(i)}=0$ for all $i$. But this is a contradiction. Thus $Q^{(i)}$ is an atom for all $i$. Then in particular, $Q_{v}^{(1)}$ $=P_{\nu} \rightarrow Q^{(1)}=P$, completing the proof.

Theorem 19. $G$ is closed.

Proof. It must be shown that any sequence $\left(E_{\nu}\right)$ of $E_{\nu} \in G$ convergent in $B^{\prime}$ converges to an element $E$ of $G: E_{v} \rightarrow E$. If $d_{v}$ denotes the maximal number of pairwise orthogonal atoms of $E_{v}, d$ denotes the corresponding number for $E$, then $d_{v} \rightarrow d$ is to be shown. That is there exist only finitely many $d_{v} \neq d$ : For each $E_{v}$ choose a system of orthogonal atoms $Q_{\nu}^{(i)}$ with $\sum_{i=1}^{d_{v}} Q_{\nu}^{(i)}=E_{v}$. Put $Q_{\nu}^{(i)}=0$ for $i>d_{\nu}$. In case of need, we can manage by selecting subsequences that all $\left(Q_{\nu}^{(i)}\right)$ converge to $Q^{(i)}: Q_{\nu}^{(i)} \rightarrow Q^{(i)}$. By the preceding theorem $Q^{(i)}$ is an atom or zero. $Q^{(i)}=0$ holds exactly 
for those $i$ with only finitely many $Q_{v}^{(i)}$ distinct from zero, i.e. the sequence $\left(d_{v}\right)$ converges. Let $d$ denote the limit of $\left(d_{v}\right)$. Then $Q^{(i)}=0$ for $i>d, Q^{(i)} \neq 0$ for $i \leqq d$. Thus $E_{v}=\sum_{i=1}^{n} Q_{\nu}^{(i)} \rightarrow \sum_{i=1}^{n} Q^{(i)}=\sum_{i=1}^{d} Q^{(i)}$.

Since $\mu\left(V, E_{\nu}\right) \leqq 1$ for all $V \in K$, so $\sum_{i=1}^{d} Q^{(i)} \leqq \mathbf{1}$ and hence $Q^{(i)} \perp Q^{(j)}$ for $i \neq j$. Therefore there holds $\sum_{i=1}^{d} Q^{(i)}=E \in G$ and because of the modularity of $G d$ is maximal. This completes the proof.

In two subsequent papers by Mr. DÄHN and Mr. SToLz further structures will be pointed out and that first without using axiom 4 and second with using axiom 4 . Finally it results that with axiom 4 an irreducible system ([5], p. 343) can be represented in this manner: $K$ is the set of all positive, semidefinite, Hermitean operators $V$ on a finite-dimensional Hilbert-space (over the fields of either the real or the complex numbers or the quaternions), $V$ satisfying $\operatorname{Tr}(V)=1 . \hat{L}$ is the set of all positive, semidefinite Hermitean operators $F \leqq 1 . \mu(V, F)$ is given by $\mu(V, F)$ $=\operatorname{Tr}(V \cdot F)$.

I thank Dr. DäHN very much for perusing and translating the manuscript, him and Mr. SToLz for a critical perusal of the proofs.

\section{References}

1. LudwiG, G.: Hauptsätze über das Messen als Grundlage der Hilbert-Raumstruktur der Quantenmechanik. Z. Naturforsch. 22a, 1303-1323 (1967).

2. - Ein weiterer Hauptsatz über das Messen als Grundlage der Hilbert-Raumstruktur der Quantenmechanik. Z. Naturforsch. 22a, 1324-1327 (1967).

3. - English preprint combining [1] and [2].

4. - Versuch einer axiomatischen Grundlegung der Quantenmechanik und allgemeinerer physikalischer Theorien. Z. Physik 181, 233-260 (1964).

5. - Attempt of an axiomatic foundation of quantum mechanics and more general theories, II. Commun. Math. Phys. 4, 331-348 (1967).

6. Bourbaki, N.: Espaces vectoriels topologiques, chap. II, deuxième édition. Paris: Hermann 1966.

7. Rose, G.: Zur Orthomodularität von Wahrscheinlichkeitsfeldern. Z. Physik 181, 331-332 (1964).

8. Brrkhoff, G.: Lattice Theory. Amer. Math. Soc. Colloq. Publ., 25, revised (1948).

GüNTHER LUDwig

Institut für Theoretische Physik (I)

der Universität

3550 Marburg, Renthof 7 